\title{
Esophageal tuberculosis with Esophagopulmonary fistula: A case report
}

\author{
Akshay Rawat ${ }^{1}$, Anurag Govil ${ }^{2}$, Dinesh Agrawal ${ }^{3}$, Harsh Udawat ${ }^{3}$, Sandeep Vaishnav ${ }^{3}$ \\ From ${ }^{1}$ Senior Resident, ${ }^{2}$ Head, ${ }^{3}$ Consultant, Department of Gastroenterology, Santokba Durlabhji Memorial Hospital, Jaipur, Rajasthan, India
}

\begin{abstract}
Esophageal tuberculosis (TB) is a rare manifestation of tuberculosis with secondary involvement being more common. The most common presentation is with dysphagia and a high index of suspicion for a tubercular etiology should be kept in mind, especially in a TB endemic region like India. Early diagnosis is essential to prevent complications such as bleeding, perforation, or stricture and also because the disease is curable with antitubercular therapy. We present a case report of a young female who presented to our hospital with complaints of cough and dysphagia and was subsequently diagnosed with esophageal tuberculosis complicated by an esophagopulmonary fistula.
\end{abstract}

Key words: Esophagus, Fistula, Tuberculosis

$\mathrm{E}$ sophageal tuberculosis (TB) is a rare condition that accounts for only $2.8 \%$ of all cases of gastrointestinal TB [1]. It usually occurs as a result of direct spread from mediastinal lymph nodes and rarely from the lungs or bloodstream. Two types of esophageal TB have been described: Primary and secondary [2]. The secondary type is more common as primary involvement is extremely rare due to the intrinsic protective properties of the esophagus [3]. If left untreated, esophageal TB can lead to bleeding, perforation, fistula formation, aspiration pneumonia, fatal hematemesis, traction diverticula, and esophageal strictures [4]. Thus, through this case report, we wish to emphasize that it is imperative to keep esophageal $\mathrm{TB}$ as an important differential diagnosis, particularly in cases of dysphagia [5].

\section{CASE REPORT}

A 27-year-old female presented to our tertiary care center with complaints of cough (especially after eating), dysphagia, decreased appetite, and undocumented weight loss (in form of loosening of clothes) for the past one month. She denied any history of fever, hemoptysis, hematemesis, nasal regurgitation, hoarseness or change in voice, dyspnea, or any chest pain.

Pallor was present but the rest of the physical examination was unremarkable. Pulse rate was $96 / \mathrm{min}$ with a regular rhythm and no radioradial or radiofemoral delay, respiratory rate was $22 / \mathrm{min}$, blood pressure was $122 / 86 \mathrm{mmHg}$ in the right arm in the supine position, and weighed $52 \mathrm{~kg}$ on admission.

\section{Access this article online}

Received - 10 May 2021

Initial Review - 26 May 2021

Accepted - 14 June 2021

DOI: $10.32677 / I J C R .2021 . v 07.106 .012$
Routine biochemical tests revealed that she had microcytic, hypochromic anemia with hemoglobin of $7 \mathrm{~g} / \mathrm{dl}$ (normal range: $12-15.5 \mathrm{~g} / \mathrm{dl}$ ), mean corpuscular volume (MCV) of 74.9 (80-95 fl), mean corpuscular hemoglobin $(\mathrm{MCH})$ of 23.7 (27.5-33.2 pg), and serum iron of $15(60-170 \mu \mathrm{g} / \mathrm{dl})$ with total iron-binding capacity (TIBC) of $160(240-450 \mu \mathrm{g} / \mathrm{dl})$. Total leukocyte counts were $9.09\left(4.5-11 \times 10^{9} / 1\right)$ and platelet count of $294\left(150-400 \times 10^{9} / 1\right)$. The liver function tests and renal function tests were within normal limits. Human immunodeficiency virus (HIV) serology was negative and sputum staining for acid-fast bacillus (AFB) was also negative.

$\mathrm{X}$-ray chest showed a homogenous opacity in the right upper zone and ultrasonography (USG) of the abdomen was suggestive of bilateral increased renal parenchymal echogenicity. In view of her complaints of dysphagia, an upper gastrointestinal endoscopy was planned which showed a deep ulcerated friable area at around $21 \mathrm{~cm}$ from upper incisors along with a possible fistulous opening at its base (Fig. 1). However, there was no significant esophageal luminal narrowing and multiple biopsies were taken from ulcer margins and sent for histopathological examination and tuberculosis-polymerase chain reaction (TB-PCR) analysis. A computed tomography (CT) thorax done thereafter showed a cavitating consolidation in the posterior segment of the right upper lobe with fistulous communication to the esophagus (Fig. 2a) along with multiple bilateral miliary lung nodules (Fig. 2b) and small mediastinal non-necrotic lymph nodes. Esophageal biopsy was suggestive of a chronic granulomatous inflammatory process with multiple ill-defined collections of epithelioid cells in the submucosa along with a few Langhan's type giant cells, but neither caseation nor any AFB could be demonstrated. The Mantoux test was positive but the TB-PCR was negative.

Correspondence to: Dr. Akshay Rawat, Room no 101, B-80 Sona Enclave, Rajinder Marg, Jaipur, Rajasthan, India. E-mail: akshayrawat89@gmail.com

(C) 2021 Creative Commons Attribution-NonCommercial 4.0 International License (CC BY-NC-ND 4.0). 


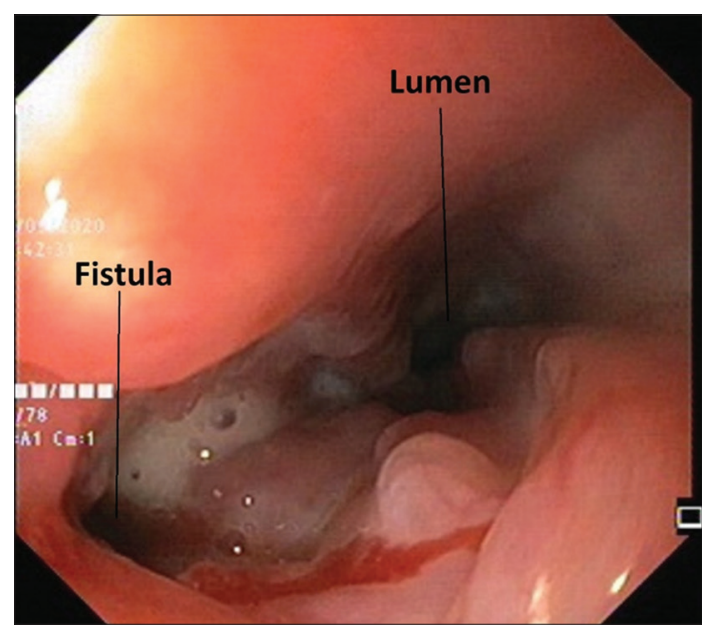

Figure 1: Endoscopic image of esophagus showing the friable, ulcerated, nodular area with fistula, and the patent esophageal lumen

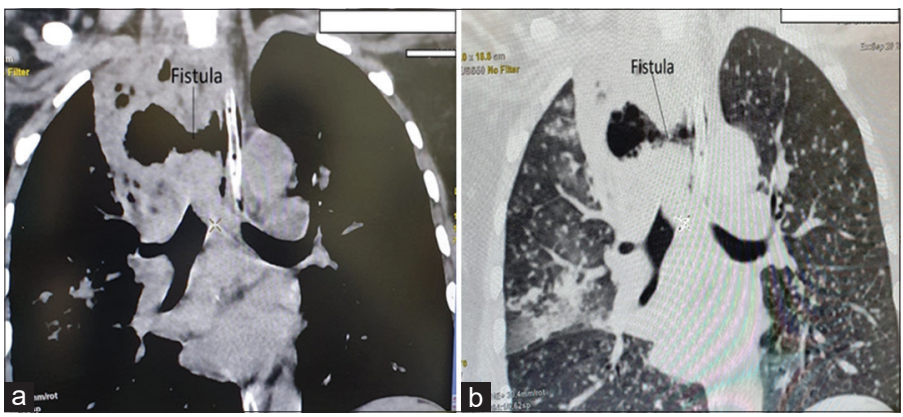

Figure 2: (a) Coronal CT image of thorax showing the (a) fistulous communication between the esophagus and the right upper lobe cavitary lesion; (b) fistulous communication along with the miliary lung lesions and right upper lobe cavitating consolidation

After multidisciplinary consultation with pathologist, radiologist, pulmonologist, and reviewing the clinical evidence (history, endoscopic, histopathology, and imaging) available, it was finally decided to treat her for probable tubercular infection with antitubercular therapy (ATT). For the esophageal fistula, an initial conservative treatment trial with nasogastric tube feeding to bypass the fistula and provide adequate nutrition was planned along with ATT. The patient and her family members were counseled that in case of persistence of the fistula, despite 4 weeks of conservative treatment, either a self-expanding metal stent or surgery could be attempted.

\section{DISCUSSION}

The overall prevalence of esophageal TB is very rare. This has been attributed to the rapid clearance of esophageal contents during swallowing. It has been shown to be present in up to $0.5 \%$ of the patients that present with dysphagia [6]. Esophageal involvement by TB usually occurs in the middle third of the esophagus at the level of the carina [7]. This is usually caused by direct extension and spread from mediastinal structures; other mechanisms include spreading from inoculation of swallowed sputum, as well as hematogenous or lymphatic spread.

Dysphagia is the most common presenting symptom, but odynophagia and retrosternal pain have also been reported $[1,8]$.
Other symptoms include low-grade fever, malaise, decrease in appetite, and weight loss [1,5]. Clinical, radiological, and endoscopic features of esophageal TB are not well-defined because of its rarity and also its close resemblance with other symptomatic esophageal disorders [1]. Approximately $65 \%$ of the patients with esophageal TB have non-specific findings on chest radiograph; however, CT chest can show characteristic pulmonary or caseating tuberculous lymphadenitis [4]. In severe cases where a trachea-esophageal fistula has formed, complaints of choking on swallowing and aspiration pneumonia can be found [9]. Thus, the non-specific presentation combined with the rarity of the disease and limited evidence in the existing literature means that esophageal TB can be misdiagnosed (e.g., as esophageal cancer) and may lead to unnecessary interventions [10].

Endoscopy and, nowadays, endoscopic ultrasound (EUS) have facilitated the early diagnosis of esophageal TB [11]. EUS is an important modality for diagnosis, which is especially useful in cases of extrinsic bulge lesions, as it helps to identify the exact layer of the esophageal wall. The lesion is located in or in patients with mediastinal lymphadenopathy wherein EUS-guided fine-needle aspiration (FNA) samples can be extremely helpful. Similarly, in cases with submucosal bulges, EUS-guided FNA is preferred over normal endoscopic biopsy because of its higher sensitivity [12].

Histopathology (HPE) report and TB-PCR remain investigations of choice in reaching the diagnosis of esophageal TB [13]. However, the sensitivity of identifying typical caseating granulomas on histopathology of endoscopy samples is low, ranging from $25.0 \%$ to $60.8 \%$. This is because of either low density of tuberculous granulomas in affected tissue or insufficient representation of the submucosal layer, the main site of tuberculous granulomas, on endoscopic biopsies [14]. Ziehl-Neelsen staining for AFB of the biopsy sample is another option but has lower sensitivity [9]. Considering histopathology as the gold standard, PCR has $74.1 \%$ sensitivity and $96.1 \%$ specificity [15]. The main differential diagnoses are esophageal cancer and Crohn's disease of the esophagus, both having completely different management options than esophageal TB [16].

Esophageal involvement does not affect the treatment, and ATT is highly effective. Most of the patients respond well to ATT [17]. Surgery is required only in cases of complications such as fistulas, strictures, and perforations. There are cases where treatment was successfully carried out with only three drugs, excluding pyrazinamide, for 6 months [18]

\section{CONCLUSION}

Tubercular involvement of the esophagus is uncommon with dysphagia being the most common presenting feature. With the advent of endoscopic techniques and EUS, diagnosis is now easier to make and this condition should be considered as a differential diagnosis whenever a lesion or even a stricture is negative for malignancy. There needs to be an increased index of suspicion among patients with dysphagia in TB endemic regions. Histopathology and TB-PCR are the key to confirm the diagnosis. 
Delay in the diagnosis can lead to complications, which might require surgical intervention; otherwise, this condition is effectively treated with ATT.

\section{REFERENCES}

1. Patel N, Amarapurkar D, Agal S, Baijal R, Kulshrestha P, Pramanik S, et al. Gastrointestinal luminal tuberculosis: Establishing the diagnosis. J Gastroenterol Hepatol 2004;19:1240-6.

2. Carr DT, Spain DM. Tuberculosis in a carcinoma of the esophagus. Am Rev Tuberc 1942;46:346-9.

3. Fahmy AR, Guindi R, Farid A. Tuberculosis of the oesophagus. Thorax 1969;24:254-6.

4. Nagi B, Lal A, Kochhar R, Bhasin DK, Gulati M, Suri S, et al. Imaging of esophageal tuberculosis: A review of 23 cases. Acta Radiol 2003;44:329-33.

5. Mou Y, Zeng H, Wang QM, Yi H, Liu W, Wen D, et al. Esophageal tuberculosis initially misdiagnosed by endoscopy as a submucosal tumor. Endoscopy 2015;47 Suppl 1:E30-1.

6. Jain SK, Jain S, Jain M, Yaduvanshi A. Esophageal tuberculosis: Is it so rare? Report of 12 cases and review of the literature. Am J Gastroenterol 2002;97:287-91.

7. Huang YK, Wu YC, Liu YH, Liu HP. Esophageal tuberculosis mimicking submucosal tumor. Interact CardioVasc Thoracic Surg 2004;3:274-76.

8. Anees A, Singh KD, Khan MA, Khan S. Tubercular esophagocutaneous fistula: A rare case. Int J Health Allied Sci 2017;6:53.

9. Hu Z, Wang X, Gong L, Zhou Y, Meng M, Feng H, et al. Case report esophageal tuberculosis presenting as a submucosal tumor. Int J Clin Exp Med 2018;11:1226-32.
10. Tang Y, Shi W, Sun X, Xi W. Endoscopic ultrasound in diagnosis of esophageal tuberculosis: 10-year experience at a tertiary care center. Dis Esophagus 2017;30:1-6.

11. Gordon AH, Marshall JB. Esophageal tuberculosis: Definite diagnosis by endoscopy. Am J Gastroenterol 1990;85:174-7.

12. Han XM, Yang JM, Xu LH, Nie LM, Zhao ZS. Endoscopic ultrasonography in esophageal tuberculosis. Endoscopy 2008;40:701-2.

13. Khanna V, Kumar A, Alexander N, Surendran P. A case report on esophageal tuberculosis a rare entity. Int J Surg Case Rep 2017;35:41-3.

14. Welzel TM, Kawan T, Bohle W, Richter GM, Bosse A, Zoller WG. An unusual cause of dysphagia: Esophageal tuberculosis. J Gastrointestin Liver Dis 2010;19:321-4.

15. Chawla K, Gupta S, Mukhopadhyay C, Rao PS, Bhat SS. PCR for $M$. tuberculosis in tissue samples. J Infect Dev Ctries 2009;3:83-7.

16. Griga T, Duchna HW, Orth M, Nicolas V, Müller KM, SchultzeWerninghaus $\mathrm{G}$, et al. Tuberculous involvement of the esophagus with oesophagobroncheal fistula. Digest Liver Dis 2002;34:528-31.

17. Baijal R, Agal S, Amarapukar DN, Kumar HR, Kotli N, Jain M. Esophageal tuberculosis: An analysis of fourteen cases. J Dig Endosc 2010;1:14-8.

18. Preto PJ, Gouveia A, Fonseca E, Pimenta M. Mediastinal tuberculous lymphadenitis presenting as an esophageal intramural tumor: A very rare but important cause for dysphagia. World J Gastroenterol 2007;13:6104-8.

Funding: None; Conflicts of Interest: None Stated.

How to cite this article: Rawat A, Govil A, Agrawal D, Udawat H, Vaishnav S. Esophageal tuberculosis with Esophagopulmonary fistula: A case report. Indian J Case Reports. 2021;7(6):257-259. 